\title{
VISUALIZATION OF COVARIANCE AND CROSS-COVARIANCE FIELDS
}

\author{
Chao Yang, ${ }^{1}$ Dongbin Xiu, ${ }^{2}$ \& Robert M. Kirby ${ }^{1, *}$ \\ ${ }^{1}$ School of Computing, University of Utah, Salt Lake City, UT 84112, USA \\ ${ }^{2}$ Department of Mathematics, Purdue University, West Lafayette, IN 47907 \\ Original Manuscript Submitted: 04/14/2011; Final Draft Received: 07/27/2011
}

\begin{abstract}
We present a numerical technique to visualize covariance and cross-covariance fields of a stochastic simulation. The method is local in the sense that it demonstrates the covariance structure of the solution at a point with its neighboring locations. When coupled with an efficient stochastic simulation solver, our framework allows one to effectively concurrently visualize both the mean and (cross-)covariance information for two-dimensional (spatial) simulation results. Most importantly, the visualization provides the scientist a means to identify the interesting correlation structure of the solution field. The mathematical setup is discussed, along with several examples to demonstrate the efficacy of this approach.
\end{abstract}

KEY WORDS: visualization, covariance, cross-covariance, generalized polynomial chaos, stochastic Galerkin methods, stochastic collocation methods

\section{INTRODUCTION}

In the past two decades, there has been a tremendous growth of interest within the computational science and engineering (CS\&E) community concerning the topics of validation and verification $(\mathrm{V} \& \mathrm{~V})$ and uncertainty quantification (UQ) in the context of numerical simulation results. With the advent of such UQ computational techniques as the stochastic finite element method [1] and generalized Polynomial Chaos method [2], there is an increasing need to convey UQ results in concise, informative ways. Visualization is the lens often through which scientists investigate their data. In response to the surge of UQ focus within the simulation community, uncertainty visualization is considered one of the top visualization research problems by the scientific visualization community [3]. In this paper, we provide a numerical technique and corresponding visualization methodology for visualizing covariance and cross-covariance fields derived from stochastic simulation data. Our framework allows one to effectively visualize both the mean and covariance information concurrently for two-dimensional (spatial) simulation results. From this, the corresponding visual representations allow the scientist a means of identifying interesting correlation structure within the solution field.

\subsection{Motivation}

Our work was originally motivated by a bioengineering application in which we were employing the gPC Galerkin approach to understand the impact of variations in organ conductivity on the classic bioelectric electrocardiograph (ECG) forward problem [4]. In that study, the conductivity of the lungs (and other various organ groups) was considered to be expressed by a collection of independent uniform random variables. Two-dimensional forward ECG simulations were conducted, and the corresponding mean and standard deviation fields were displayed. In Fig. 1, we provide example flood-contour visualizations as presented in [4].

${ }^{*}$ Correspond to Robert M. Kirby, E-mail: kirby@cs.utah.edu

2152-5080/13/\$35.00 C 2013 by Begell House, Inc. 


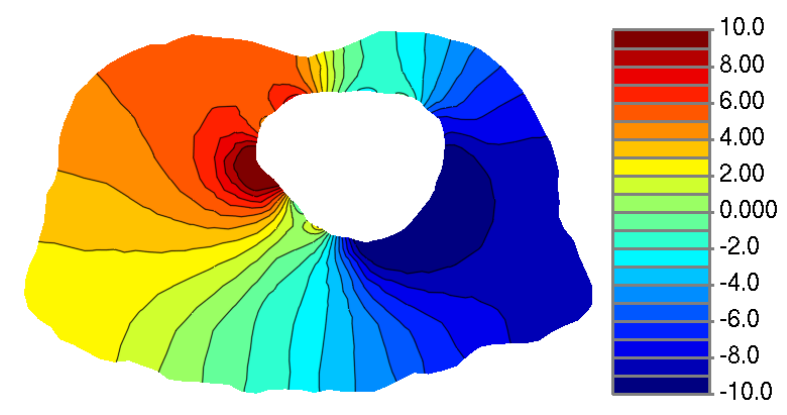

(a)

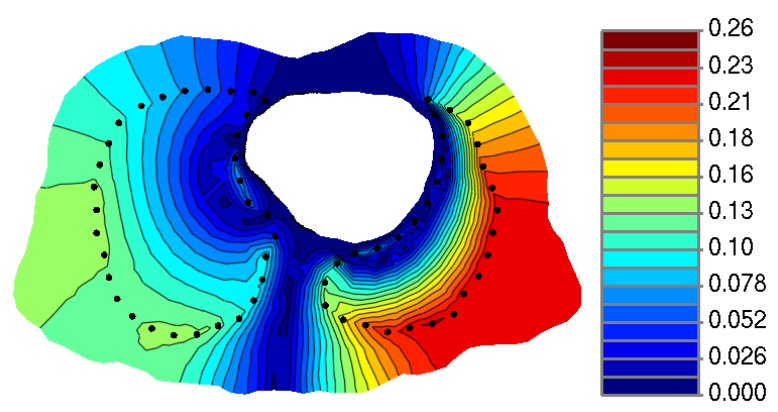

(b)

FIG. 1: Effects of stochastic variation in conductivities of the lungs on torso potentials: These contour plots correspond to the stochastic behavior of the electrical potential across a slice through the torso resulting from stochastic organ conductivities. (a) Shows the mean for a stochastic interval of $\pm 50 \%$ from the reference lung conductivity, while (b) shows the associated standard deviation, in which the region of varying conductivity is indicated by the region enclosed by the dotted contours. All units in the plot are millivolts.

Although plotting the mean and standard deviation in separate plots has been the standard way to visualize the results, practitioners, when presented such plots, prefer a way to overlay the two visualizations for concurrent examination and, more importantly, a way to visualize the correlation structure of the field to fully assimilate the significance of the results with respect to the underlying practical problem. This, along with the experience of the authors on this topic, motivated the current work, which is to find a means of simultaneously portraying both mean and (cross-) covariance information in one visualization.

\subsection{Related Work}

The field of uncertainty visualization, though still in its early development, is not new. Simultaneous with the two decades of UQ simulation research, there has also been a host of visualization research projects aimed at providing corresponding UQ visualization techniques. For example, as early as 1996, three uncertainty visualization results surface within visualization community. (1) Lodha et al. [5] proposed a number of methods to visualize uncertainty; (2) Wittenbrink et al. [6] proposed a new vector glyph to show both the magnitude and direction of uncertainty; and (3) Pang et al. [7] broadly surveyed different kinds of uncertainty visualization methods and provide a formal classification of uncertainty visualization. The work of Pang et al.'s [7] is regarded as one of the most comprehensive surveys of current uncertainty visualization methodologies to date.

Here we briefly summarize the aforementioned work of Pang et al.'s [7]. In this work, uncertainty is classified into three categories: statistical, error, and range. The different forms of uncertainty are introduced into the pipeline as data are acquired, transformed, and visualized. They propose a classification on uncertainty visualization with five characteristics: (1) value of datum and its associated value uncertainty (scalar, vector, tensor, multivariate), (2) location of datum and its associated positional uncertainty (0D, 1D, 2D, 3D, time, etc.), (3) extent of datum location and value (discrete or continuous), (4) visualization extent (discrete or continuous), and (5) axes mapping defining visualization mapping (experiential or abstract). They then develop a number of new uncertainty visualization methods and organize them based on how the uncertainty information is encoded into seven categories: add glyphs, add geometry, modify geometry, and modify attributes, animation, sonification, and psychovisual approaches.

With the original work of Pang et al.'s [7] in place, a diverse number of further contributions sprang forth. For example, Johnson and Sanderson [8] discussed the need for uncertainty visualization in the scientific imaging field. Typically, error and uncertainty of data are included as 2D graphs but left out of two-dimensional and three-dimensional visualizations. The expression of error or uncertainty can be made by various techniques (depending on the application), such as glyphs, which can either be new glyphs specific for conveying error, or by altering the size and orientation of current glyphs using error or uncertainty as a variable. Also, adding new visual cues to the visualization 
can be used as a way to convey uncertainty: bump maps, blurring, textures, and other lighting attributes, for example, can be used, taking advantage of the fact that these techniques may distract the eye, thus giving a cue that there are problems/uncertainties in these areas.

Further research has been accomplished on visual attributes used to visualize uncertainty. Djurcilov et al. [9] introduced uncertainty in volume rendering by using pseudo-color, adding noise, and altering texture. Rhodes et al. [10] presented uncertainty in isosurface by visualizing uncertainty with hue, brightness, and saturation. Grigoryan and Rheingans [11] introduced point-based primitives to show surface uncertainty by displacing individual surface points along its normal by an amount proportional to a random number multiplied with the local uncertainty value, hence creating a "fuzzy surface" to convey the surface uncertainty. Hengl [12] described GIS methods for visualization of uncertainty based on the hue-saturation-intensity (hue, saturation, and intensity), color model and calculations with colors using the color mixture (CM) concept. Hengl and Toomanian [13] illustrated how color mixing and pixel mixing can be used to visualize uncertainty to represent prediction error in soil property maps. MacEachren et al. [14] reviewed and assessed progress toward visual tools and methods to help analysts manage and understand information uncertainty. They conclude, by identifying seven key research challenges in visualizing information uncertainty, particularly as it applies to decision making and analysis.

There is some research on using texture as a technique for uncertainty visualization. Interrante [15] introduced textures to visualize spatial uncertainty by distorting regularity in natural textures. Sanderson et al. [16] created a method for visualizing vector fields while potentially presenting uncertainty by using a reaction-diffusion model to generate texture patterns with variable shapes, sizes, and orientations. Botchen et al. [17] proposed two texture-based techniques to visualize uncertainty in time-dependent two-dimensional flow fields. The first method employed a cross advection perpendicular to the flow direction. The second method applied isotropic diffusion that implemented by Gaussian filtering. They compare these techniques with an adapted multifrequency noise approach.

Cedilnik and Rheingans [18] presented procedurally-generated annotation by distortion that indicates uncertainty. The authors proposed how procedural variation of width, sharpness, noise, and amplitude modulation can indicate uncertainty. The method presented here is to use annotations that are generated procedurally and slightly distorted in areas of high uncertainty. The distortion techniques should procedurally create the annotation reasonably fast that are perceptually normalized and inherently meaningful. They stated that their method preserves perceptibility across various levels of uncertainty.

Olston and Mackinlay [19] proposed the bounded visualization method that should be different from statistical uncertainty, because statistical uncertainty representations potentially incorporate an infinite ranges of values. They proposed "ambiguation" (i.e., purposeful ambiguity) to visually conveyed ambiguous regions in two-dimensional graphs and charts. Aigner et al. [20] proposed a new glyph, called PlanningLines to show temporal uncertainty. Love et al. [21] developed a system to visualize multivalued field including uncertainty. Kniss et al. [22] developed a volume visualization method that incorporates the fuzzy classification into the visualization cycle to allow users to interactively explore the uncertainty, risk, and probabilistic decision of surface boundaries.

There is also considerable research on uncertainty visualization being accomplished within the various application domains. Schmidt et al. [23] developed visual representations for real multidimensional sea-bottom uncertainty data, multivariate information, and multidimensional target state data by using of coloring methods, surface representations, glyphs, haptics, iconographic imagery, animation, and text. Lundstrom et al. [24] proposed a probabilistic animation method to visualize uncertainty in medical volume renderings. First, they have a probabilistic transfer function model, which allows for direct user interaction with the classification. It is animated by sampling the probability domain over time, which results in varying appearance for uncertain regions. Zuk and Carpendale [25] presented a theoretical analysis of uncertainty visualization in which they evaluated eight uncertainty visualizations from various sources on widely accepted visualization principles and showed a set of heuristics and how pertinent each heuristic was with respect to the sampled visualizations. Janoos et al. [26] proposed a surface representation of neurons method to reconstruct dendrites and their spins from 3D microscopy imaging. They identify the valid spin and the classification the spins based on their morphology in a Bayesian fashion. They employed the pseudocolor to visualize the uncertainty of the data. A user study was conducted to evaluate the visualization. They concluded that visualization techniques that distort the shape of the object were not suitable in a clinical setting. They added too much visual clutter while not conveying any additional information. Correa et al. [27] presented a framework using statistics methods, such as

Volume 3, Number 1, 2013 
uncertainty modeling, propagation, and aggregation in the visual analytics process. They adapted data transformations such as regression, principal component analysis (PCA), and K-means clustering to account for uncertainty. They followed common data analysis tools, such as model fitting, principal component analysis, and clustering to show uncertainty is both propagated and aggregated in these stages on data from the Boston neighborhood housing price data set. They then presented the visual representations that combines summarized and detailed views of the uncertainty of multidimensional data.

Visualization of correlation and covariance matrices has been also explored in the literature. Braak [28] extended the biplot technique, which visualizes correlations between pairs of variables and regression coefficients to canonical correlation analysis and redundancy analysis. Friendly [29] proposed a method called "Corrgram" based on two main schemes: (1) rendering the value of a correlation to depict its sign and magnitude and (2) reordering the variables in a correlation matrix so that "similar" variables are positioned adjacently, facilitating perception. Baker [30] put visualization techniques into practice to explore covariance in the inverse ocean modeling framework. The paper introduced a glyph-based method called "covariance neighborhoods" to show local covariance. The glyphs produced, however, differ from those presented in this work. Gunawardane et al. [31] introduced an integration of statistical analysis that computes correlation and linear regression between indicators using time-series data with visualization techniques to gain deeper socio-economic understanding. The technique we present here supplements this list of methods and represents a new (cross)-covariance visualization and glyph-based icon generation strategy.

\subsection{Outline}

The paper is organized as follows. In Section 2, we lay out the mathematical details of the work. In Section 3, we present the implementation details necessary to replicate this work. In Section 4, we present our new methodology applied to two different stochastic data sets, one involving multiple random variables over a rectangular, structured spatial mesh and one involving the original motivating problem, simulation results of electric potential over a twodimensional torso slice. We summarize our results in Section 5.

\section{MATHEMATICAL DESCRIPTION}

\subsection{Preliminaries}

Here, we consider a physical domain $D$ in two dimensions, that is, $x=\left(x_{1}, x_{2}\right) \in D \subset \mathbb{R}^{2}$, and a stochastic solution field $u(x, \omega)$, where $\omega \in \Omega$ in a properly defined probability event space $\Omega$. The statistical moments of the solution field that will be considered in this paper include the mean and variance fields,

$$
\mu_{u}(x)=\mathbb{E}[u(x, \omega)], \quad \sigma_{u}^{2}(x)=\mathbb{E}\left[\left(u-\mu_{u}\right)^{2}\right]
$$

respectively. The covariance field is a function of two spatial locations $x$ and $y$,

$$
C_{u u}(x, y)=\mathbb{E}\left\{\left[u(x)-\mu_{u}(x)\right]\left[u(y)-\mu_{u}(y)\right]\right\} .
$$

Note $C_{u u}(x, x)=\sigma_{u}^{2}(x)$. The cross-covariance field between two solution fields $u$ and $v$ is

$$
C_{u v}(x, y)=\mathbb{E}\left\{\left[u(x)-\mu_{u}(x)\right]\left[v(y)-\mu_{v}(y)\right]\right\} .
$$

A useful quantity to reflect the relation between the two fields is the cross-correlation coefficient

$$
\operatorname{cov}_{u v}(x, y)=\frac{C_{u v}(x, y)}{\sigma_{u}(x) \sigma_{v}(y)}
$$

From its definition, $-1 \leq \operatorname{cov}_{u v}(x, y) \leq 1$.

Our goal is to construct a technique to visual the (cross-)covariance field effectively. 


\subsection{Local Cross-Covariance Field}

Both the covariance (COV) and cross-covariance (xCOV) fields are functions of two spatial locations. Consequently, it is nontrivial to visualize them in a standard coordinate system. To circumvent this difficulty, we define a localized version of these fields.

Definition 1: Local cross-covariance field: For a given location $\hat{x}$ and a real parameter $\epsilon \geq 0$, the local crosscovariance of two stochastic fields $u(x, \omega)$ and $v(x, \omega)$ with respect to a given location $\hat{x}$ is defined as

$$
C_{u v}^{\epsilon}(\hat{x}, y)=\mathbb{E}\left\{\left[u(\hat{x})-\mu_{u}(\hat{x})\right]\left[v(y)-\mu_{v}(y)\right]\right\}
$$

where $y$ resides on the $\epsilon$-ball surrounding $\hat{x}$, i.e.,

$$
\|y-\hat{x}\|=\epsilon
$$

where $\|\cdot\|$ is the standard Cartesian norm. For a single stochastic field $u(x, \omega)$, the definition of the local covariance field naturally becomes

$$
C_{u u}^{\epsilon}(\hat{x}, y)=\mathbb{E}\left\{\left[u(\hat{x})-\mu_{u}(\hat{x})\right]\left[u(y)-\mu_{u}(y)\right]\right\}
$$

It is easy to see that as the radius of the $\epsilon$-ball decreases, the local covariance field becomes the variance at $\hat{x}$, i.e.,

$$
\lim _{\epsilon \rightarrow 0} C_{u u}^{\epsilon}(\hat{x}, y)=\sigma_{u}^{2}(\hat{x})
$$

These local fields are more amenable to the standard visualization technique because they are functions of the single variable $y$ that surrounds a fixed location $\hat{x}$.

\subsection{Local Cross-Covariance Shape}

On the basis of the definition of local cross-covariance, we can now define a geometric shape to reflect its structure.

Definition 2: Local cross-covariance shape: For the local cross-covariance field $C_{u v}^{\epsilon}$ with respect to a fixed location $\hat{x}$, as defined in (5), the local cross-covariance shape $S_{u v}$ is defined as a closed curve $z(s) \in \mathbb{R}^{2}$ surrounding $\hat{x}$, where $s$ is a parametrization, such that for all $s$ satisfying $\|y(s)-\hat{x}\|=\epsilon$,

$$
\|z(s)-\hat{x}\|=\left|C_{u v}^{\epsilon}[\hat{x}, y(s)]\right|
$$

For a single stochastic field $u(x, \omega)$, this leads to the local covariance shape $S_{u u}$,

$$
S_{u u}=\left\{z|\|z(s)-\hat{x}\|=| C_{u u}^{\epsilon}(\hat{x}, y(s)) \mid, \forall s:\|y(s)-\hat{x}\|=\epsilon\right\}
$$

where $C_{u u}^{\epsilon}(\hat{x}, y)$ is the local covariance field in (7).

It is easy to see that as $\epsilon \rightarrow 0$, the local covariance shape $S_{u u}$ becomes a circle with radius $\sigma_{u}^{2}(\hat{x})$. For $\epsilon>0$, the shape can be considered as a deformed circle, where the radius at any angle is determined by the (absolute) value of (cross-)covariance field at the same angle.

\section{IMPLEMENTATION DETAILS}

We now discuss the implementation details of visualizing the local cross-covariance. This is based on the definition of the local cross-covariance shape in Definition 2. For clarity of exposition, we discuss the covariance and crosscovariance separately, even though by our definitions the covariance can be considered a special case of the crosscovariance. 


\subsection{Stochastic Solution Fields}

The solution field of (cross-)covariance is obviously required in order to perform visualization. In practice, however, rarely does one compute the (cross-)covariance field directly, for the dimensionality of the field is excessively large. Instead, what one usually has is the solution field itself. Here, we will assume that the solution fields $u(x, \omega)$ and $v(x, \omega)$ are known approximately via numerical simulation. (For the covariance field, the second solution field $v$ is not required.)

For a stochastic solution field, one of the widely adopted approximations is via the generalized polynomial chaos framework [1, 2]. In this approach, the solution dependence in the random space is approximated by a set of orthogonal polynomial bases. A typical gPC approximation takes the following form:

$$
u(x, \omega) \approx \sum_{|\mathbf{i}|=0}^{N} \widetilde{u}_{\mathbf{i}}(x) \Phi_{\mathbf{i}}[Z(\omega)]
$$

where $\mathbf{i}=\left(i_{1}, \ldots, i_{d}\right)$ is multi-index of length $d \geq 1$ with $\left|\mathbf{i}=i_{1}+\cdots+i_{d}\right|$ and $Z(\omega) \in \mathbb{R}^{d}$ is a random vector of dimension $d$ representing the random inputs. The dimensionality $d$ is determined by the nature of the random inputs of the stochastic simulation. $\left\{\Phi_{\mathbf{i}}(Z)\right\}$ is a set of orthogonal polynomials satisfying

$$
\mathbb{E}\left[\Phi_{\mathbf{i}} \Phi_{\mathbf{j}}\right]=\delta_{\mathbf{i}, \mathbf{j}}
$$

where $\delta_{\mathbf{i}, \mathbf{j}}=\delta_{i_{1}, j_{1}}, \ldots, \delta_{i_{d}, j_{d}}$ is the $d$-variate Kronecker $\delta$ function, whose value is 1 if $\mathbf{i}=\mathbf{j}$ and 0 otherwise.

When the solution field is approximated in this form, the solution statistics can be readily evaluated in a postprocessing step. For example, the mean of the solution is

$$
\mu_{u}(x) \approx \widetilde{u}_{0}(x)
$$

and the covariance is

$$
C_{u u}(x, y) \approx \sum_{|\mathbf{i}|>0}^{N} \widetilde{u}_{\mathbf{i}}(x) \widetilde{u}_{\mathbf{i}}(y) .
$$

When another field $v(x, \omega)$ is approximated in a similar way, the cross-covariance can be approximated as

$$
C_{u v}(x, y) \approx \sum_{|\mathbf{i}|>0}^{N} \widetilde{u}_{\mathbf{i}}(x) \widetilde{v}_{\mathbf{i}}(y)
$$

Similar expression for the covariance $C_{u u}(x, y)$ can be obtained by replacing the second field $v$ by $u$. We remark that there exists a variety of gPC-type expansions, using basis functions other than the orthogonal polynomials in Eq. (12). A detailed discussion on this is beyond the scope of this paper. We only emphasize here that, regardless of the type of the basis, such expansions always allow one to evaluate the solution statistics in the postprocessing step without incurring any simulation effort.

The expansion coefficients in Eq. (11) are usually solved by a set of deterministic equations deriving from the governing stochastic system, via either the stochastic Galerkin or the stochastic collocation approach. (Details of these approaches can be found in [32].) We assume that a proper spatial discretization (e.g., finite elements, finite difference, etc.) is employed for these deterministic equations so that the expansion coefficients are now approximated by a set of spatial basis functions-usually piecewise polynomials. By doing so, Eq. (11) can be determined in any spatial location by mere numerical evaluation. Consequently, the solution statistics of the underlying stochastic field can be approximated by evaluating its gPC expression at any spatial location, without resorting to simulations. 


\subsection{Local Covariance Shape}

Again let us consider a fixed location $\hat{x}$ and the local covariance field defined in (7). Let us also fix the constant $\epsilon$ at a positive value. We now discretize the $\epsilon$-ball along its angular direction. For $n>1$, let

$$
\theta^{j}=(j-1) \cdot 2 \pi / n, \quad j=1, \ldots, n
$$

and

$$
y_{1}^{j}=\hat{x}_{1}+\epsilon \cos \left(\theta^{j}\right), \quad y_{2}^{j}=\hat{x}_{2}+\epsilon \sin \left(\theta^{j}\right) .
$$

Consequently, $\left\{y^{j}\right\}_{j=1}^{n}$ is a set of discrete points on the $\epsilon$-ball surrounding $\hat{x}$. We remark that it is not mandatory to use equal-distanced points in the angular direction.

The local covariance can now be computed on these points; that is,

$$
C_{u u}^{j}(\hat{x}) \triangleq C_{u u}\left(\hat{x}, y^{j}\right)=\mathbb{E}\left\{\left[u(\hat{x})-\mu_{u}(\hat{x})\right]\left[u\left(y^{j}\right)-\mu_{u}\left(y^{j}\right)\right]\right\}, \quad j=1, \ldots, n .
$$

We now normalize these values by setting

$$
C_{u u}^{j}(\hat{x}) \leftarrow \frac{C_{u u}^{j}(\hat{x})}{\left\|\mathbf{c}_{u u}(\hat{x})\right\|_{p}}
$$

where $\mathbf{c}_{u u}=\left(C_{u u}^{1}, \ldots, C_{u u}^{n}\right)$ is a vector and $\|\cdot\|_{p}$ is the standard vector $p$-norm. (Other vector norms can be readily employed.)

The covariance shape is now represented discretely by the following points, $z^{j}$, for $j=1, \ldots, n$, where

$$
z_{1}^{j}=\hat{x}_{1}+\alpha \cdot\left|C_{u u}^{j}(\hat{x})\right| \cos \left(\theta^{j}\right), \quad z_{2}^{j}=\hat{x}_{2}+\alpha \cdot\left|C_{u u}^{j}(\hat{x})\right| \sin \left(\theta^{j}\right) .
$$

Here, the constant factor

$$
\alpha=\gamma \sigma_{u}^{2}(\hat{x})
$$

for a real constant $\gamma>0$.

Note that in the setup of the $\epsilon$-ball, if $\epsilon \rightarrow 0$, then $C_{u u}(\hat{x}) \rightarrow \sigma_{u}^{2}(\hat{x})$. Subsequently, $C_{u u}^{j}$ becomes constant 1 for all $j$, and the points $\left\{z^{j}\right\}$ now lie on a circle of radius $\gamma \sigma_{u}^{2}(\hat{x})$. The presence of the constant factor $\gamma>0$ is for scaling purpose. One should experiment with the value of $\gamma$ so that the size of the circle results in an effective visualization (e.g., avoiding overlapping icons, etc.).

To make a distinction between positive and negative covariance values, we propose to use different line patterns when rendering the shape. For example, one can use a solid line on the part of the shape where $C_{u u}>0$ and a dotted line where $C_{u u}<0$. Other choices are certainly possible. For example, one may choose to use different coloring to distinguish the positive and negative parts. We remark that it is necessary to identify the location $\hat{x}$ in the rendering so that the distortion of the base circle is more visible. In Fig. 2, we present a diagram demonstrating various constituents of the local covariance field icon.

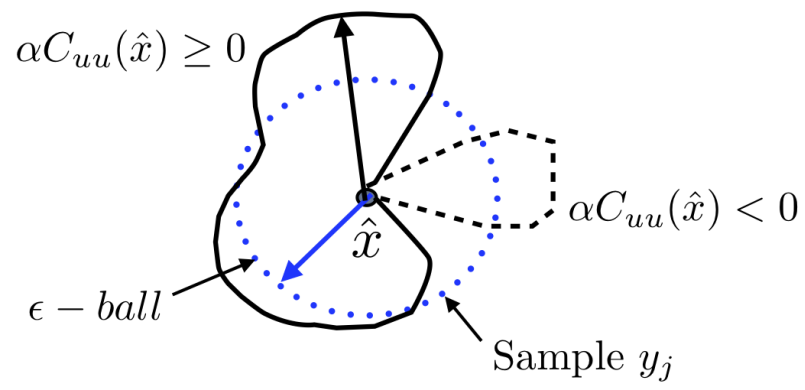

FIG. 2: Diagram presenting the constituents of the local covariance field icon. The various symbols used in the diagram are explained in the text.

Volume 3, Number 1, 2013 
We also remark that when rendering the covariance shape, one is not required to use the same set of discretization points for computing the $\epsilon$-ball. Another type of discretization can be employed for smooth rendering of the shape. In such a case, some type of interpolation is required to evaluate the local covariance field at the location of $z$. Owing to the nature of visualization, it is not necessary to use an extremely high-order interpolation. A relatively low-order local interpolation scheme would be sufficient for efficient implementation.

\subsection{Local Cross-Covariance Shape}

For the local cross-covariance field between two stochastic field $u$ and $v$, the above procedure for local covariance field is essentially repeated, with the difference that the quantities corresponding to $C_{u u}$ are now replaced by those corresponding to $C_{u v}$. In short, we first compute

$$
C_{u v}^{j}(\hat{x}) \triangleq C_{u v}\left(\hat{x}, y^{j}\right)=\mathbb{E}\left[\left(u(\hat{x})-\mu_{u}(\hat{x})\right)\left(v\left(y^{j}\right)-\mu_{v}\left(y^{j}\right)\right)\right], \quad j=1, \ldots, n
$$

and then normalize the result by setting

$$
C_{u v}^{j}(\hat{x}) \leftarrow \frac{C_{u v}^{j}(\hat{x})}{\left\|\mathbf{c}_{u v}(\hat{x})\right\|_{p}}
$$

where $\mathbf{c}_{u v}=\left(C_{u v}^{1}, \ldots, C_{u v}^{n}\right)$.

The local cross-covariance shape is now represented by a set of discrete points, similar to the case of local covariance shape in (20), as follows:

$$
z_{1}^{j}=\hat{x}_{1}+\alpha \cdot\left|C_{u v}^{j}(\hat{x})\right| \cos \left(\theta^{j}\right), \quad z_{2}^{j}=\hat{x}_{2}+\alpha \cdot\left|C_{u v}^{j}(\hat{x})\right| \sin \left(\theta^{j}\right)
$$

where

$$
\alpha=\gamma \cdot C_{u v}(\hat{x}, \hat{x})
$$

for a scaling constant $\gamma>0$. It is obvious that when the two fields $u$ and $v$ become the same field $u$, then the above rendering of the local cross-covariance shape will become the same as the local covariance shape described in the previous section. Once again, we propose to use different line patterns, or coloring, on the shape in the positive and negative portion of $C_{u v}(\hat{x})$.

A slight variation exists, when one is more interested in understanding the cross-correlation coefficients (4), for these are normalized quantities and may be more revealing than the actual cross-covariance field. In this case, we can define the factor $\alpha$ as, instead of Eq. (25),

$$
\alpha=\gamma \cdot\left|\operatorname{cov}_{u v}(\hat{x}, \hat{x})\right| \text {. }
$$

Again, one can use different line patterns or coloring to distinguish the positive and negative portions of the shape. By doing so, the base area of the shapes will correspond to the $\operatorname{cov}_{u v}$ value, which is scaled by the standard deviations $\sigma_{u}$ and $\sigma_{v}$. Consequently, the influence of the standard deviations is eliminated. In the limiting case of $\epsilon \rightarrow 0$, the $\operatorname{cov}= \pm 1$, where the sign depends on the correlation. The local shape thus becomes circles with a constant radius $\gamma$ in the entire domain, and each circle is either completely positive or completely negative. Though not quite interesting, if this limiting case is what one desires to visualize, then it is not necessary to plot the circles (for they are the same radius). One can simply allow $\alpha=0$ and use different symbols at $\hat{x}$ to denote the positive and negative signs of the covariance.

\subsection{A Short Remark on $\epsilon$}

In all the discussions, the role of $\epsilon$ is important. From the definition of the local cross-covariance (5) and covariance (7), the parameter $\epsilon$ signifies how "local" the quantity is, with the limiting case of $\epsilon \rightarrow 0$ giving the local pointwise (cross-)covariance at the chosen point $\hat{x}$. In practice, to understand how the (cross-)covariance function varies away from $\hat{x}$, one can choose to produce a set of visualizations, each using different values of $\epsilon$. Note that the (cross)covariance function often settles to a constant value when two physical locations are "far" away, where "far" here is relative to the (cross-)correlation length. Thus, it is the intermediate values of $\epsilon$ that are more revealing to examine. Such intermediate values are relative to the (cross-)correlation length and are problem dependent. 


\section{RESULTS}

In this section, we present two simulation examples of our proposed (cross)-covariance icon visualization methodology. The first example involves an elliptic computation on a rectangular domain using a Chebyshev collocation method in space and gPC stochastic collocation to represent the stochastic variation. The second example involves the solution of the elliptic bioelectric forward problem on an unstructured triangular finite element mesh in which traditional linear finite elements are used for the discretization in space and gPC stochastic collocation is used to represent the stochastic variation. Note the underlying stochastic problem and solution technique is not very important in our demonstration. The visualization requires only the solution fields expressed by finite element approximation and the gPC approximation.

Because the mathematical methodology presented previously extends trivially to vectors of independently distributed random variables and to cross-correlation of multiple fields, we limit our results to only demonstrating icons for the correlation structure.

\subsection{D Elliptic Solution on a Rectangular Domain}

This example is based on the simulation results of [33, Section 4.2]. It is a simulation of an elliptic problem in a random domain, where the random domain was transformed into a fixed domain and the governing elliptic problem becomes a stochastic elliptic equation. The transformed (and fixed domain) is a rectangle of $5 \times 1$ units, with 25 and 7 Chebyshev collocation points per direction, respectively. The random space has 10 random dimensions, which are required to model the uncertainty of the bottom boundary. The gPC Galerkin method was employed with third-order expansion. Details of the simulation are in [33].

In Fig. 3 we present flood contours of the mean of the solution (top) and the variance in the solution (bottom) due to randomness in the bottom boundary. These plots represent what would traditionally be presented: two separate images for mean and variance, and were what were presented in [33].

In Fig. 4 we present flood contours of the mean of the solution with variance icons generated based on a collection of samples within the data (denoted by circular dots). The variance icons have been scaled so that they are nonoverlapping. Note again that because we are dealing with a field expressed in both finite basis in physical space and gPC in random space, we can sample the mean and variance anywhere and are not explicitly constrained to only sample the solution at the original collocation points. This allows us a flexibility in terms of the locations at which we place the icons. In this example, a uniform lattice sampling is used.

In Fig. 5, we represent flood contour plots of the mean of the solution with covariance icons generated using $\epsilon=0.01$ (top) and $\epsilon=0.1$ (bottom). Icons are drawn based on 20 local points around each location, and spline interpolation is used to form the icon shape centered around the sample points. Icons are omitted if the $\epsilon$-ball needed for their generation does not lie completely within the computational domain. For the very small $\epsilon$ value $(\epsilon=0.01)$,
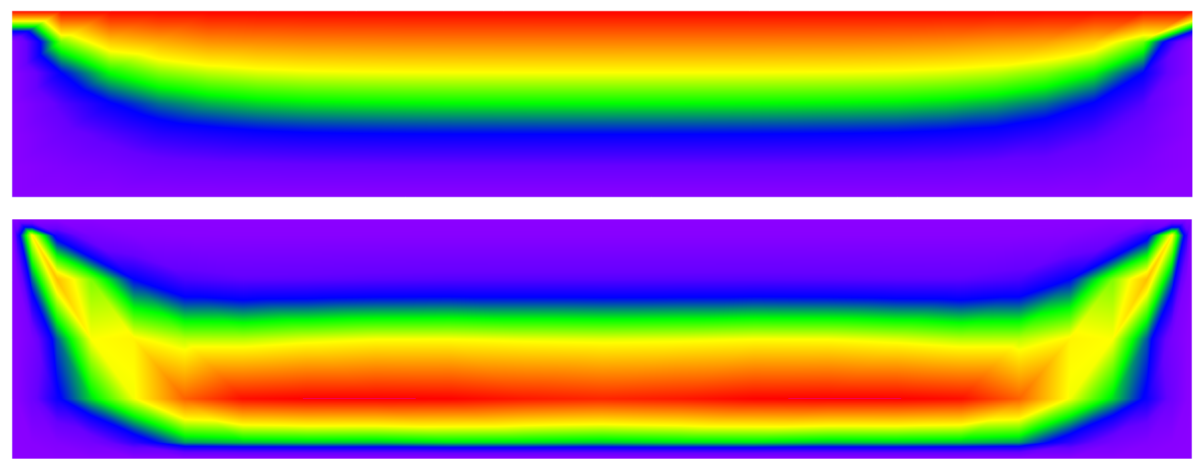

FIG. 3: Flood contours showing the mean of the solution (top) and the variance in the solution (bottom) of the 2D rectangular domain elliptical system due to uniform variation of the lower boundary height plotted in the traditional way, as two separate images. 


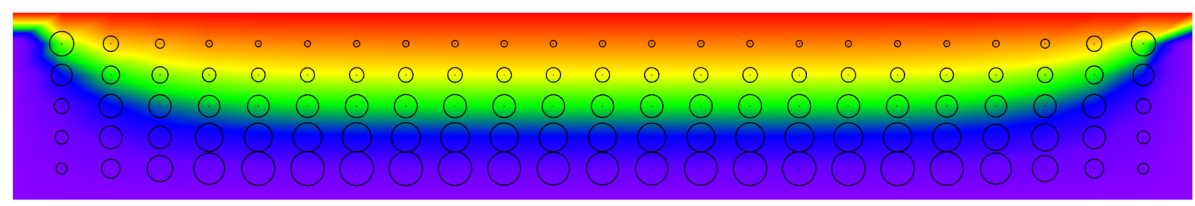

FIG. 4: Flood contour of the mean of the solution with variance icons due to uniform variation of the lower boundary height.
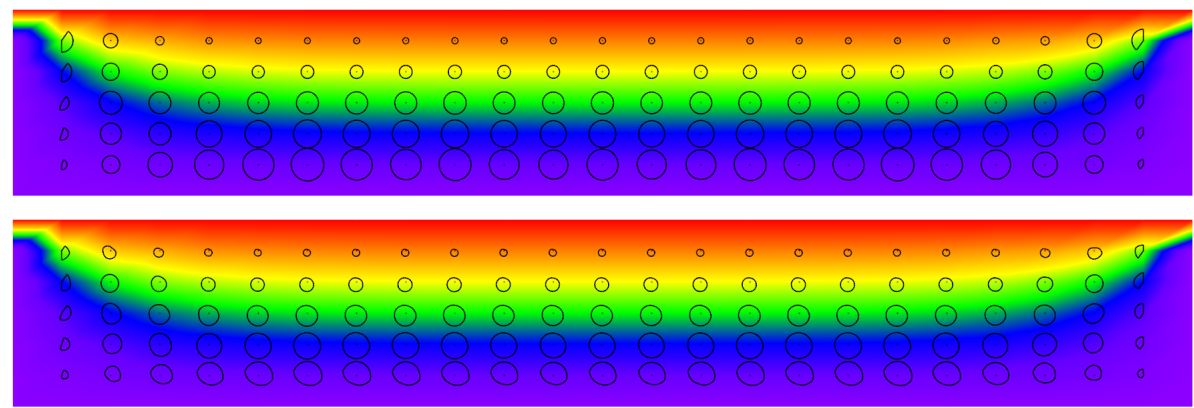

FIG. 5: Flood contour of the mean of the solution with covariance icons due to uniform variation of the lower boundary height. Top: Icons generated using $\epsilon=0.01$; Bottom: Icons generated using $\epsilon=0.1$. Note that icons are omitted if the $\epsilon$-ball needed for their generation does not lie completely within the computational domain.

the icons are almost all circles, for this is close to the limiting case of $\epsilon \rightarrow 0$. For larger value of $\epsilon=0.1$, the distortion is obvious near the bottom of the domain, indicating interesting covariance structure that is worth investigating by the simulation scientists. Such indications of regions of further enquiry are what visualization should deliver.

\subsection{D Bioelectric Problem Solved on a Unstructured Domain}

This example is based on the data set used in our motivating example taken from [4] in which we were interested in solving the bioelectric forward problem. The data set consists of a triangular finite element method obtained through the segmentation of MRI data. There are 618 vertices and 1071 triangles in the computational mesh. To replicate the results in [4] (which employed the gPC Galerkin approach), the gPC collocation approach was used with nine quadrature points in the stochastic direction. Only perturbations with respect to a single uniformly-distributed random variable are considered. In Fig. 6, we present the mean of the electric potential (top) and the variance (bottom) in potential due to a $\pm 50 \%$ variation in the lung conductivities. These plots represent what would traditionally be pre-

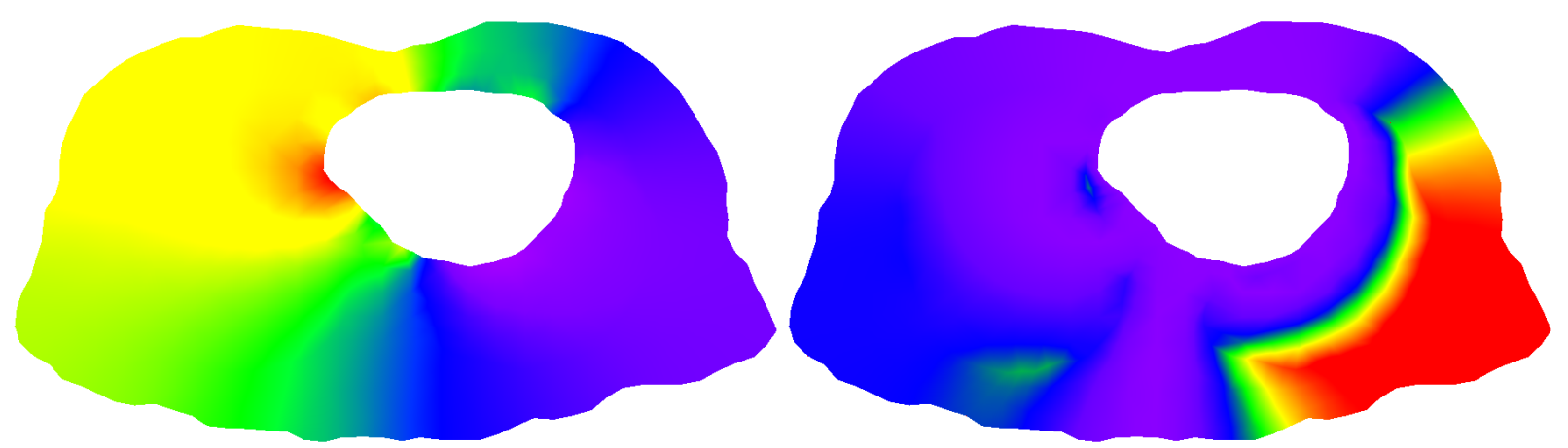

FIG. 6: Mean of the potential field (left) and variance in the potential field due to $\pm 50 \%$ uniform variation in the lung conductivity (right) plotted in the traditional way, as two separate images. 
sented: two separate images for mean and variance. We repeat the images here for consistency of the color-map in all images will compare.

In Fig. 7 we present flood contours of the mean of the electric potential with variance icons (left) and with covariance icons (right). In the case of the variance icons, the variance is computed at a variety of points (denoted with circular dots within the image) and the corresponding variance at those points is used as the radius of the circle having the sample point as its center. The variance icons have been scaled so that they are nonoverlapping. For the covariance plot, the covariance of the field with itself is computed (i.e., $C_{u u}$ ) at a collection of sample points (again denoted with circular dots) with an $\epsilon$-ball of 0.1 . Icons are drawn based on 60 samples around each point and spline interpolation is used to form the icon shape centered around the sample points. Regions of positive covariance are denoted with solid lines, whereas regions of negative covariance are denoted by dashed lines. Icons are omitted if the $\epsilon$-ball needed for their generation does not lie completely within the computational domain.

A quick examination of the visualization plots indicates that there are interesting correlation patterns near the corners. If Fig. 8, we present two zoom images of the covariance plot. The flood contours denote the mean of the
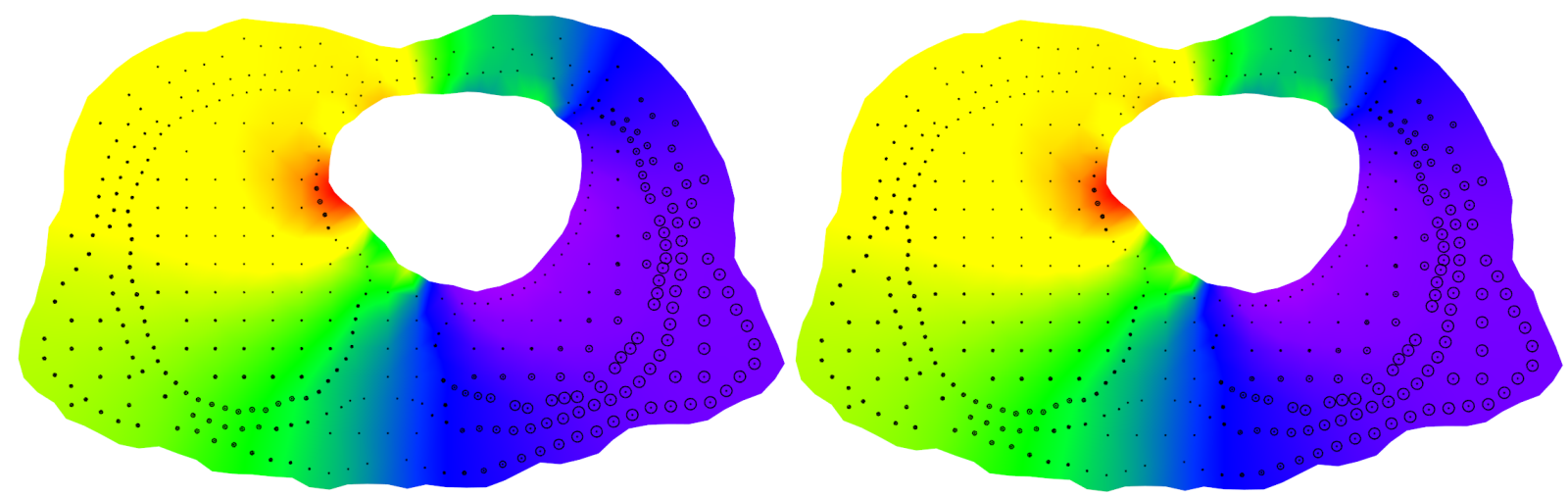

FIG. 7: Flood contour of the mean of electric potential with variance icons (left); Flood contour of the mean of the electric potential with covariance icons for $\epsilon=0.1$ (right). Variance and covariance are in the electric potential field due to $\pm 50 \%$ uniform variation in the lung conductivity. Note that icons are omitted if the $\epsilon$-ball needed for their generation does not lie completely within the computational domain.
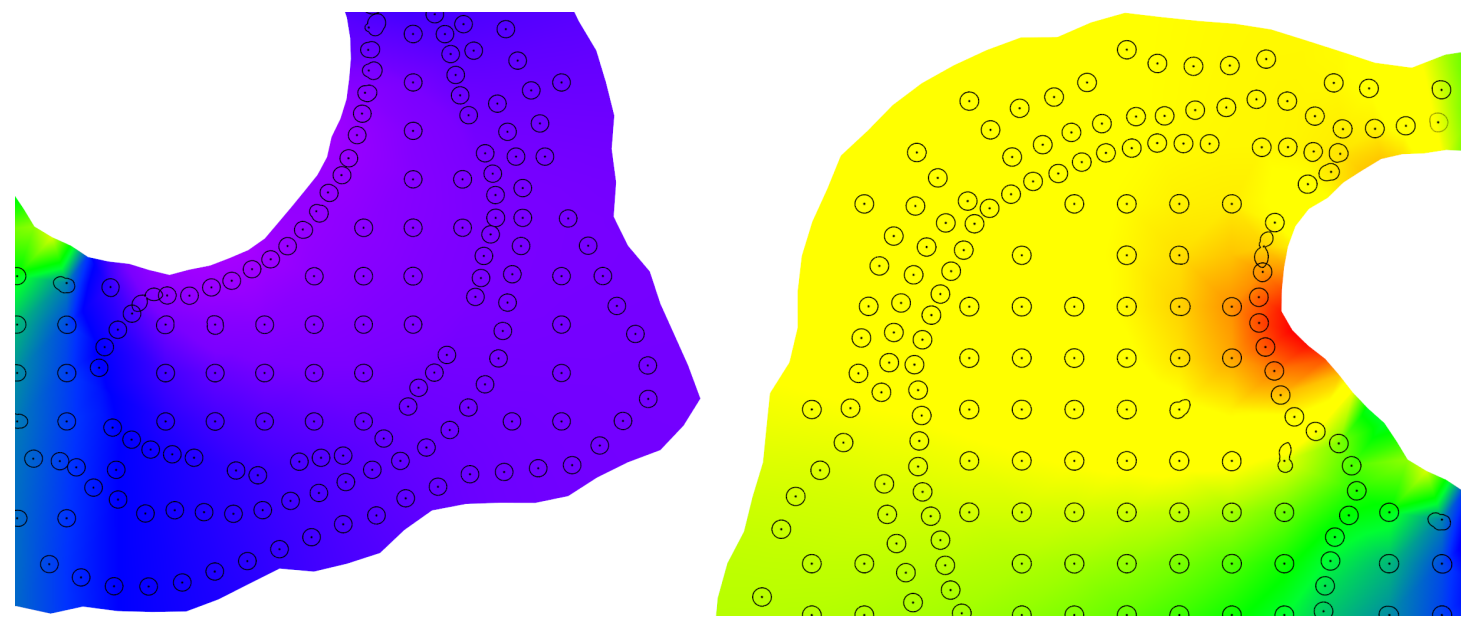

FIG. 8: Flood contour of the mean in electric potential with covariance icons for $\epsilon=0.1$. Left: Zoom of right bottom of the image; Right: Zoom of the left-top of the image. Covariance is in the electric potential field due to $\pm 50 \%$ uniform variation in the lung conductivity. Note that icons are omitted if the $\epsilon$-ball needed for their generation does not lie completely within the computational domain. 
electric potential. Note in the the right plot one can clearly see icons that are noncircular, denoting a covariance structure that has biased directions. This kind of visualization information cannot be obtained from the standard variance contour plot presented in Fig. 6. Also note that in the right image of Fig. 8, one can clearly see dashed icons denoting covariance regions that are negative.

\section{SUMMARY}

With the continued growth of the areas of V\&V and UQ within the simulation community, there will be an ever increasing need for effective visualization methods for representing data and simulation result uncertainty. For relevant visualization techniques to be generated which serve the needs of the simulation and application domain communities, there must exist an interaction between the mathematical UQ community and the visualization community - an interaction which provides fruitful research opportunities for both.

In this paper, we present a numerical technique to visualize covariance and cross-covariance fields of two-dimensional stochastic simulation. The method was originally motivated by a bioengineering application in which the concurrent visualization of uncertainty information was desired. The method we have proposed is local in the sense that it demonstrates the covariance structure of the solution at a point with its neighboring locations. When coupled with a stochastic solution strategy, such as the generalized polynomial chaos, our framework allows one to effectively concurrently visualize both the mean and covariance information for two-dimensional (spatial) simulation results in an easy-to-understand, succinct representation. Most importantly, the visualization provides the scientist a means to identify interesting correlation structure of the solution field. The mathematical extension of this work to three-dimensional fields is straightforward; however, the many visualization issues, such as glyph occlusion, will need to be addressed in future work. This work provides an example of effective interaction between the UQ and visualization communities in attempting to solve a specific mathematical abstraction and visualization need.

\section{ACKNOWLEDGMENTS}

The authors thank Prof. Chris Johnson and Dr. Kristi Potter for discussions concerning this work. This is a collaborative research project supported under Grants No. NSF IIS-0914564 (Kirby) and NSF No. IIS-0914447 (Xiu), and through No. DOE NET DE-EE0004449 (Kirby) and No. DOE DE-SC0005713 (Xiu). Infrastructure support provided through Grants No. NSF-IIS-0751152.

\section{REFERENCES}

1. Ghanem, R. G. and Spanos, P., Stochastic Finite Elements: A Spectral Approach, Springer-Verlag, New York, NY, 1991.

2. Xiu, D. B. and Karniadakis, G. E., The Wiener-Askey polynomial chaos for stochastic differential equations, SIAM J. Sci. Comput., 24:619-644, 2002.

3. Johnson, C., Top scientific visualization research problems, IEEE Comput. Graphics Appl., 24(4):13-17, 2004.

4. Geneser, S. E., MacLeod, R. S., and Kirby, R. M., Application of stochastic finite element methods to study the sensitivity of ECG forward modeling to organ conductivity, IEEE J. Biomed. Eng., 55(1):31-40, 2008.

5. Lodha, S., Wilson, C., and Sheehan, R., Listen: Sounding uncertainty visualization, Proceedings of IEEE Visualization 1996, pp. 189-195, Nov. 1996.

6. Wittenbrink, C., Pang, A., and Lodha, S., Glyphs for visualizing uncertainty in vector fields, IEEE Trans. Vis. Comput. Graphics, 2(3):266-279, 1996.

7. Pang, A. T., Wittenbrink, C. M., and Lodh, S. K., Approaches to uncertainty visualization, The Vis. Comput., 13:370-390, 1996.

8. Johnson, C. and Sanderson, A., A next step: Visualizing errors and uncertainty, IEEE Comput. Graphics Appl., 23(5):6-10, 2003. 
9. Djurcilov, S., Kim, K., Lermusiaux, P. F. J., and Pang, A., Volume rendering data with uncertainty information, Data Visualization 2001, Proceedings of the Joint EUROGRAPHICS_IEEE TCVG Symposium on Visualization, Springer-Verlag, Berlin, pp. 243-252, 2001.

10. Rhodes, P. J., Laramee, R. S., Bergeron, R. D., and Sparr, T. M., Uncertainty visualization methods in isosurface volume rendering, Eurographics 2003, Short Papers, pp. 83-88, 2003.

11. Grigoryan, G. and Rheingans, P., Probabilistic surfaces: Point based primitives to show surface uncertainty, Proceedings of IEEE Visualization 2002, pp. 147-153, 2002.

12. Hengl, T., Visualisation of uncertainty using the his colour model: Computations with colours, Proceedings of 7 th International Conference on GeoComputation, pp. 8-17, 2003.

13. Hengl, T. and Toomanian, N., Maps are not what they seem: Representing uncertainty in soil property maps, Proceedings of 7th International Symposium on Spatial Accuracy Assessment in Natural Resources and Environmental Sciences, pp. 805-813, 2006.

14. Maceachren, A. M., Robinson, A., Gardner, S., Murray, R., Gahegan, M., and Hetzler, E., Visualizing geospatial information uncertainty: What we know and what we need to know, Cartogr. Geogr. Inf. Sci., 32:139-160, 2005.

15. Interrante, V., Harnessing natural textures for multivariate visualization, IEEE Comput. Graphics Appl., 20(6):6-11, 2000.

16. Sanderson, A. R., Johnson, C. R., and Kirby, R. M., Display of vector fields using a reaction-diffusion model, Proceedings of Conference on IEEE Visualization 2004, IEEE Computer Society, Washington, DC, pp. 115-122, 2004.

17. Botchen, R., Weiskopf, D., and Ertl, T., Texture-based visualization of uncertainty in flow fields, Proceedings of IEEE Visualization 2005, pp. 647-654, Oct. 2005.

18. Cedilnik, A. and Rheingans, P., Procedural rendering of uncertainty information, Proceedings of Conference on Visualization 2000, IEEE Computer Society Press, Los Alamitos, CA, pp. 77-84, 2000.

19. Olston, C. and Mackinlay, J., Visualizing data with bounded uncertainty, Proceedings of IEEE Symposium on Information Visualization, Boston, pp. 37-40, Oct. 2002.

20. Aigner, W., Miksch, S., Thurnher, B., and Biffl, S., Planninglines: Novel glyphs for representing temporal uncertainties and their evaluation, Proceedings of International Conference on Information Visualisation, pp. 6-8, 2005.

21. Love, A. L., Pang, A., and Kao, D. L., Visualizing spatial multivalue data, IEEE Comput. Graph. Appl., 25:69-79, 2005.

22. Kniss, J., Van Uitert, R., Stephens, A., Li, G.-S., Tasdizen, T., and Hansen, C., Statistically quantitative volume visualization, Proceedings of IEEE Visualization 2005, pp. 287-294, Oct. 2005.

23. Schmidt, G., Chen, S.-L., Bryden, A., Livingston, M., Rosenblum, L., and Osborn, B., Multidimensional visual representations for underwater environmental uncertainty, IEEE Comput. Graphics Appl., 24(5):56-65, 2004.

24. Lundstrom, C., Ljung, P., Persson, A., and Ynnerman, A., Uncertainty visualization in medical volume rendering using probabilistic animation, IEEE Trans. Vis. Comput. Graphics, 13(6):1648-1655, 2007.

25. Zuk, T. and Carpendale, S., Theoretical analysis of uncertainty visualizations, Proceedings of SPIE-IST Electronic Imaging, pp. 606-607, 2006.

26. Janoos, F., Nouansengsy, B., Xu, X., Machiraju, R., and Wong, S. T., Classification and uncertainty visualization of dendritic spines from optical microscopy imaging, Comput. Graphics Forum, 27(3):879-886, 2008.

27. Correa, C. D., Chan, Y.-H., and Ma, K.-L., A framework for uncertainty-aware visual analytics, IEEE Symposium on Visual Analytics Science and Technology, pp. 51-58, 2009.

28. Braak, C. J. F. T., Interpreting canonical correlation analysis through biplots of structural correlations and weights, Psychometrika, 55:519-531, 1990.

29. Friendly, M., Corrgrams: Exploratory displays for correlation matrices, Am. Stat., 56(4):316-324, 2002.

30. Baker, M., Visual representations of covariance for the inverse ocean modeling framework, Tech. Rep., Pervasive Technology Labs at Indiana University, Indianapolis, IN, Nov. 2006.

31. Gunawardane, P., Middleton, E., Lodha, S., Crow, B., and Davis, J., Analyzing statistical relationships between global indicators through visualization, Int. Conference on Information and Communication Technologies and Development, pp. 212-221, April 2009. 
32. Xiu, D., Numerical Methods for Stochastic Computations, Princeton Univeristy Press, Princeton, NJ, 2010.

33. Xiu, D. and Tartakovsky, D., Numerical methods for differential equations in random domain, SIAM J. Sci. Comput., 28(3):1167-1185, 2006. 